\section{Esthetic and Restorative Dentistry Material Selection and Technique}

Authors: Douglas A. Terry, Willi Geller

Publisher: Quintessenz Verlags-GmbH, Germany

Language: English

ISBN: 978-0-86715-763-5

Edition: 3/e

Publish Year: 2018

Pages: 792, illustrated

Price: $348.00 €$

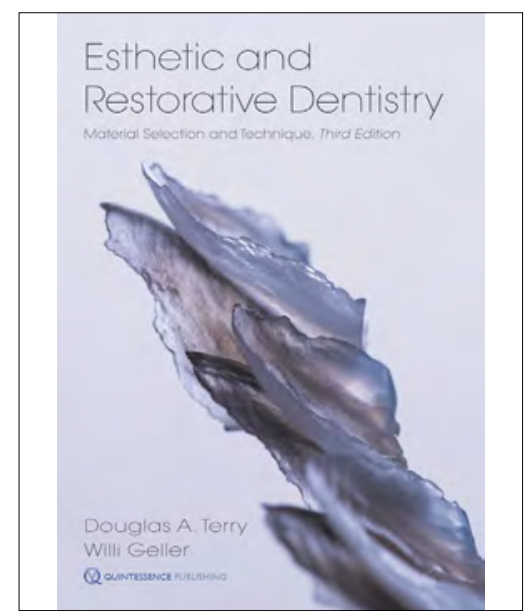

The intense and rapid development of research in dental materials and digital technologies, has made the development of new concepts for the realization of dental restorations possible.

Dr. Douglas A. Terry has become accustomed to writing high-level scientific books supplemented with clinical cases treated with the most modern aesthetic restoration methods based on an appropriate laboratory technology. His third edition of the book "Esthetic and Restorative Dentistry" is the most recent step in this evolution. The book is structured in 14 chapters and begins by establishing the diagnostic protocol based on digital photography, shade determination, diagnostic models, wax-up diagnostic and communication using eLABor_aid Concept. Further the principles of tooth preparation starting from evolution preparation design, clinical objectives of modern restorative dentistry, biomaterial selection, consideration for CAD/CAM technology and composite preparation for all five cavity classes, veneer preparation design, inlay/onlay, full coverage are presented through abundantly illustrated clinical cases. The properties and qualities of composite resins are also discussed addressing the integration, infrastructure, stress at the restorative-tooth interface, CAD/CAM resin ceramic restoration, current development in nanotechnology for each cavity class, and for diastema closure and anterior veneers. Ceramic materials also area topic of the book showing the properties, different classifications and methods. for the fabrication of a single-coping zirconia or FPD framework, and scanning methods. All ceramic crowns, feldspathic porcelain veneers, preparationless veneers, porcelain fused to metal crowns, color management of the implant supported single central incisor restorations, and balancing the esthetic zone are discussed in detail. An important stage is the impression process which is described and illustrated and includes material selection and impression techniques as well.

Mechanisms of adhesion, adhesive cements and modern interim restorative treatment concept are eloquently presented. Clinical objectives, requirements, and consideration, fabrication techniques, consideration factors in cement selection, laboratory fabrication of a composite resin FPD, direct fabrication of an anterior provisional crown, provisional veneer, indirect/direct fabrication of an implant provisional restoration and functional composite provisional are abundantly illustrated. Esthetic post systems such as direct fiber-reinforced post and core system and prefabricated fiber-reinforced post systems are discussed along this book. The authors highlight the importance of finishing and polishing esthetic restorative materials. Digital photography it is an important subject in modern dentistry and because of that an entire chapter is dedicated to this topic providing all the necessary informations like: camera system, reflective exposure measurement, guidelines for camera selection and application of digital camera systems. The end of the book brings us information about periodontal plastic surgery, including crown lengthening procedures and mucogingival surgical procedures, translating the gummy smile, and connective tissue grafting for various classes of recession type defects; interdisciplinary implantology diagnosis, assessment and treatment planning, implantation and placement and interdisciplinary surgical strategy; biomodification of tooth discoloration, based on extrinsic and intrinsic stains origin and conservative tooth correction treatment through vital and nonvital bleaching.

Dr. Douglas A. Terry, starting from the new esthetic and restorative dentistry philosophy shows us three principles for treating patients: prevention, preservation and perpetuation of longevity. He has succeeded, together with Maestro Willi Geller and his renowned referents, to accomplish an exhaustive volume through text and an exceptional over 2500 figures. This is useful book for every dentist who aims at restoring aesthetic at the highest scientific and technological level.

DOI: https://doi.org/10.25241/stomaeduj.2019.6(1).bookreview.3

The Books Review is drafted in the reviewer's sole wording and illustrates his opinions. 